\title{
MURRAY STATE

7-23-2021

\section{Effects of Parenting by Lying in Childhood on Adult Lying, Internalizing Behaviors, and Relationship Quality}

Bailey Dodd

Esther K. Malm

Murray State University, emalm@murraystate.edu

Follow this and additional works at: https://digitalcommons.murraystate.edu/faculty

Part of the Psychology Commons

\section{Recommended Citation}

Dodd, B., \& Malm, E. K. (2021). Effects of Parenting by Lying in Childhood on Adult Lying, Internalizing Behaviors, and Relationship Quality. Child Psychiatry \& Human Development, 1-8.

This Journal Article is brought to you for free and open access by the Faculty Works at Murray State's Digital Commons. It has been accepted for inclusion in Faculty \& Staff Research and Creative Activity by an authorized administrator of Murray State's Digital Commons. For more information, please contact msu.digitalcommons@murraystate.edu. 
Effects of Parenting by Lying in Childhood on Adult Lying, Internalizing Behaviors , and Relationship Quality.

Bailey Dodd and Esther K. Malm

Murray State University

\section{Author's Note}

Bailey Dodd (iD https://orcid.org/0000-0002-4045-4563

Esther Malm (iD https://orcid.org/0000-0002-5798-6128

The study was registered with Open Science Framework (OSF).The authors have no conflict of interest to disclose.

Correspondence for this article should be addressed to Dr. Esther K. Malm, Murray State University. Email: emalm@ murraystate.edu; 212 Wells Hall, Murray State University, Murray, KY 42071, USA; Tel: 270-809-6360 


\begin{abstract}
Parenting by lying is a phenomenon in which parents lie to their children, usually for a positive goal, and has been the subject of new parenting research. This study tested the associations between parenting by lying in childhood, lying to parents in young adulthood, and parent-child relationship quality. Secondly, we examined the mechanisms through which these constructs were all related to internalizing behaviors in young adulthood, specifically - stress, anxiety and depressive symptoms. Young adult participants between the ages of 18 and $24(\mathrm{~N}=$ 206 ) responded to questions about parenting strategies experienced in childhood, their current adult functioning, lying to parents, and parent-child relationship quality. Results indicate that parenting by lying in childhood was significantly associated with lower quality relationships with parents during young adulthood. Additionally, we found that double mediation models (lying to parents and quality of parent-child relationships) were best fitting the data in explaining the exploratory mechanisms (indirect pathways) through which parenting by lying was associated with internalizing behaviors (stress responses, anxiety, and depressive symptoms) in young adulthood. Based on all the findings, a review of parenting by lying in childhood as a common parenting strategy, and further research is highly encouraged. Other implications are discussed.
\end{abstract}

Keywords: Parenting by Lying, Lying to parents, Parent-child relationship quality, Stress, Anxiety, Depressive symptoms 


\section{Introduction}

Lying is a common occurrence among children and is well studied, yet research on lying by parents to their children is minimal. Parenting by lying is a phenomenon where lying is used as a form of parenting, be it intentionally or unintentionally, to influence children's actions or feelings [1]. This is an upcoming area of research with few published studies on the topic so far. Of the published studies, evidence currently indicates that parents endorse this parenting strategy for several reasons, which include to protect the feelings of their children [1], modify behaviors, and teach moral lessons through fantasy stories [2].

Parenting by lying is a very common behavior that is conventionally accepted in different cultures and is ultimately designed to shape childhood behavior into what parents identify as positive behavior (examples below). There may be various reasons why parents engage in parenting by lying, including that it is a socially accepted part of parenting, and a relatively easy parenting strategy which may be reinforced by children's own desire for rewards [1-2]. Additionally, parents in Western cultures such as the United States are more likely to lie to control their children's emotions while parents in Eastern cultures such as China find it more acceptable to lie to control their children's behaviors [2]. Children's connections with their primary caregivers and their desire for rewards may be one particular reason why parents tend to shape behavior using promises or conditions even if they are untrue [3-5]. Based on limited research so far, there are four categories of lies parents use and view as instrumental and necessary to parenting [1-2]. Based on one cross-cultural study, these four categories are: 1) lies relating to completing tasks (e.g., ' Finish all your food or you'll grow up to be short') ); 2) lies related to safety (e.g., 'If you don't follow me, a kidnapper will come to kidnap you while I'm gone"); 3) lies related to misbehavior (e.g., "If you lie to someone, your nose will grow 
longer''), and 4) lies related to children's requests (e.g., "I did not bring money with me today. We can come back another day', ) [2].

While parenting by lying may be useful in shaping childhood behavior, the potential short- and long-term negative consequences are emerging. Based on studies so far, there are possible negative impacts of parenting by lying on mental health, internalizing behaviors, and social functioning in childhood, adolescence, and adulthood. For example, Santos and colleagues [6] examined the longitudinal effects of parenting by lying in childhood on internalizing behaviors and children lying to parents in adulthood. The authors found that participants who reported higher levels of parenting by lying were more likely to report higher levels of antisocial personality problems, and lying to parents in adulthood. In turn, lying to parents was significantly associated with higher levels of internalizing behaviors, externalizing behaviors, and antisocial personality behaviors. There was also an indirect and significant relationship between parenting by lying and internalizing behaviors, via lying to parents [6]. These findings may stem from distrust and a sense of betrayal by role models over time through observed parental lying. This may also be occurring with age as children realize that the parenting strategies used to influence their behavior for a long period were (sometimes/often) lies [6].

Developmental and behavioral theories may explain the relationship between parenting by lying and the eventual tendency to lie to parents. One such theory is Bandura's social cognitive learning model [7], which suggests that young children model behaviors of their parents and role models through observation of behaviors, actions, and emotions, as well as the consequences. This theory has been well supported across a variety of parental practices (e.g., parental beliefs and styles, parental support and involvement, parent-child relationship quality) concerning child and adolescent outcomes [3, 8-12]. Further, developmental theories and studies 
have demonstrated that parents can have both positive and negative influences on their children through multiple pathways stemming from not only parents but from responses of the child(ren), and their broader environment over time [11, 13-14]. While children also play a role in choosing to lie, the process of parenting forms the foundation for social learning including lying and truth telling not only in childhood but as needed as children mature [1-2, 6].

Secondly, lying to parents (studied mostly in adolescence) is rooted in parent-child interactions. For instance, parent-child relationships, including constructs such as parent-child communication and relationship quality [15] suggest that parenting by lying shows both positive and negative effects on parent-child relationships including levels of trust and communication [15-17]. For example, in a study using data from 671 parent-child dyads, Engels and colleagues [17] found that adolescents who engaged in more lying behaviors towards their parents were significantly more likely to be secretive, disclose less, and communicate less with their parents. In addition, there was lower trust and more alienation in parent-child relationships that involved higher levels of adolescent lying [17].

\section{Purpose of Study}

This research sought to expand on the limited existing studies that investigate parenting by lying by examining the potential negative associations of parenting by lying in childhood in three related areas: lying to parents, parent-child-relationship quality, and Internalizing behaviors. First, we sought to replicate the findings of Santos et al. [6] as related to internalizing behaviors using a different sample. While the authors focused on internalizing behaviors as one broad construct, we, in this study, examine three key components of internalizing behaviors independently, namely stress responses, anxiety, and depressive symptoms as experienced in young adulthood. These three clusters of reactions/symptoms continue to be studied as separate 
conditions in extant studies and meta analytic reviews [e.g., 18], although they are sometimes treated as a construct. We wanted to know if there were differential impacts of parenting by lying, and lying to parents on stress responses (a.k.a stress in this study), anxiety, and depressive symptoms. Next, we sought to examine and understand further pathways (direct and indirect paths) through which parenting by lying and lying to parents were related to parent relationship quality, and in turn the three subcomponents of internalizing behaviors. Parent relationship quality was examined as trust, communication, alienation, and overall relationship quality [19]. While Santos and colleagues [6] found that lying to parents mediated the relationship between parenting by lying and internalizing behaviors, we propose that it is not only lying to parents but also the quality of the parent-child relationship that mediates the relationship between parenting by lying and internalizing behaviors (stress, anxiety, and depressive symptoms) in young adulthood.

We therefore hypothesized that:

1. Parenting by lying would be significantly and positively associated with internalizing behaviors (stress, anxiety, and depressive symptoms).

2. Parenting by lying would be significantly and negatively associated with (1) trust in parents, (2) communication with parents, and (3) overall parent relationship quality and (4) positively associated with parent alienation.

3. Lying to parents, in addition to parent-child relationships, would mediate the relationships between parenting by lying and stress, anxiety, and depressive symptoms.

\section{Method}

\section{Participants and Procedures}


Data for this study was collected from students from different programs who had enrolled in undergraduate psychology courses at a university in the Southern United States. Students enrolled in these courses had multiple options to complete a required research exposure component of the course with one option being research participation. For those who chose to participate in research there were many types of studies available to participate in within a span of three months. Participants who chose to partake in this study first acknowledged informed consent before taking the survey. After participants read and acknowledged consent, and voluntary participation, they received a link to the survey. The survey took approximately 25 minutes after which a debriefing statement was presented, and participants were thanked for their participation. Participants received no compensation other than course credit for completing the survey. The University's Institutional Review Board approved all study procedures (\#19-006). The exact data collection procedure and plans, in addition to all other materials (e.g., output, IRB paperwork) can be found on https://osf.io/kp6ye/files/

The sample $(\mathrm{N}=206)$ consisted of $77 \%$ females, with their ages ranging from 18 to 23 $(M=18.70$ years, $S D=0.995)$. Majority of the sample identified as White, with $4.9 \%$ African Americans, 3.9\% Hispanics, 2.9\% Asian, and 4.9\% identifying as Biracial. This ethnicity composition is representative of the region where this data was collected [20]. The final sample was adequate for this study since the minimum sample size required to detect a small effect at a power of .8 or above with six predictors was 146 (GPower v3 [21])

\section{Measures}

Parenting by Lying Scale [1]. Parenting by lying was measured through a 9-item scale of the lying scenarios. Participants were asked to rate a series of nine lies by indicating the extent to which their parents had ever told them a similar lie, using a Likert scale that ranged from 
1(absolutely no) to 7(absolutely yes). Items included six behavioral scenarios (e.g. If you go outside alone, a bogeyman will get you) and three emotional scenarios (e.g. A child is told, 'you did a good job at cleaning up your room' after making things messier). Responses to items on the parenting by lying scale were averaged, and the scores ranged between 1 and 6.78 , with higher scores indicating higher levels of parenting by lying. The original study reported acceptable reliability scores $(\alpha=0.70)$ with good internal consistency [6]. The Cronbach alpha for the scale in this study was $\alpha=0.81$.

Lying to Parents scale. The frequency at which participants lied to their parents in adulthood was measured using a 12-item questionnaire created by Engels et al. [17]. The scale asked participants to rate on a Likert scale, which ranged from 1(never) to 5 (very often,) how often they engaged in certain lying behaviors towards their parents. The 12 lie items included explicit lies to parents (e.g. Lie to your parents about the things that you engage in), white lies (e.g. Tell a white (small) lie to your parents), and lies designed to make information more interesting (e.g. Exaggerate to you parents about the things you experience). The scores of the items in the lying to parents scale were averaged, ranging from 1 to 5, with higher scores indicating higher levels of lying to parents. Similar to previous studies that have reported very high internal consistency e.g. $[6,17]$, reliability was high for this scale in the current study $(\alpha=$ $0.92)$.

Internalizing Behaviors. Internalizing Behaviors were measured using a short form of the Depression Anxiety Stress scale (DASS [22]). The short version of DASS consists of 21 items, with 7 items each on depressive (e.g., "I couldn't seem to experience any positive feeling at all”), anxiety (e.g., "I experienced trembling [e.g. in the hands]) and stress symptoms (e.g., I found it difficult to relax"). Participants were asked how applicable items were to them over the 
last week using a scale that ranged from 0 (Did not apply to me at all) to 3 (Applied to me very much or most of the time), with higher scores indicating higher levels of endorsement. Stressful response scale ranged from 7 to 28 , anxiety ranged from 6 to 24 and depressive symptoms ranged from 6 to 24 . The DASS scale has been extensively used in different populations and age groups, and reports high reliability and validity across the three subscales [22]. For the current study, Cronbach alphas for depressive, anxiety, and stress symptoms were $0.92,0.89$, and 0.86 respectively.

Relationship Quality and Trust in Others (IPPA-R; [19]). Quality of relationships, and trust in parents was measured using the IPPA-R, which consists of 28 items focused on parental relationship. The scales required the participants to read a series of scenarios and rate the degree to which they applied using the choices 0 (Never True), 1 (Sometimes True), and 2 (Always True). The IPPA-R includes three subscales for trust (e.g., "I trust my friends"), communication (e.g., "My friends listen to my opinions"), and alienation (e.g., "I feel angry with my parents").

Previous research $[19,23]$ show moderate to high validity and reliability scores. For example, the average internal consistencies IPPA-R across all three dimensions was $\alpha=0.82$. For this study, Cronbach alphas for the parent subscales were $\alpha=0.92$ for trust, $\alpha=0.89$ for communication, and $\alpha=0.86$ for alienation. In addition to the three subscales/dimensions for parent factors, an overall parent relationship quality measure was created. The two relationship quality scores were estimated by reversing scores on the alienation scale and adding them to scores on the trust and communication scales. Cronbach alphas for this study was 0.92 for overall parent relationship quality.

\section{Data Analyses}


Data was analyzed using SPSS v23 with the mediation software add-on PROCESS. Data was cleaned, and a series of bivariate Pearson's r correlations were conducted, along with basic descriptive information, among each of the major variables prior to regression analyses of the hypotheses. To address hypothesis three, we ran exploratory double mediation models, to examine the pathways through which lying to parents and the four parent-child relationship quality variables facilitated the relationship between parenting by lying and internalizing behaviors (depressive, anxiety, and stress symptoms). These double meditations were run using SPSS Process Macros v3.1 with bootstrapping and 95\% Bias corrected bootstrapped confidence interval reported [24]. As the data are cross-sectional, we refer to these mediation analyses as exploratory mediation analyses.

\section{Results}

\section{Preliminary Analyses:}

Using SPSS v23, correlational analyses and linear regressions were conducted to measure the associations between parenting by lying, lying to parents, adult functioning, and parent relationships. Parenting by lying and lying to parents were significantly associated with other variables. Parenting by lying, lying to parents, and parent-child relationship variables were all significantly associated with depressive, anxiety, and stress symptoms in the expected directions.

In addition, this study had more females than males as participants, thus independent samples t-tests were run to determine if there were significant mean differences between the groups (males versus females) on the outcome variables. Results showed that there was no significant group difference on anxiety $(\mathrm{t}(204)=1.22, p=.23)$ and depression scores $(\mathrm{t}(204)=-$ $.57, p=.57)$, however, there was for stress responses, where females reported more stress than males $(\mathrm{t}(204)=2.05$, Mean difference $=1.63, p=.04)$. Thus, sex was entered as a covariate for mediations related to stress as the outcome. 
Descriptive statistics and correlations for each variable are outlined in Table 1. Next, linear regressions and mediation analyses were conducted to examine the three hypotheses.

Internalizing behaviors. Parenting by lying was significantly associated with lying to parents $(\beta=0.23, p<0.001)$. With regards to hypothesis 1 , higher levels of parenting by lying were found to be associated with higher levels of $(1)$ stress $(\beta=0.29, p<0.001),(2)$ anxiety $(\beta=$ $0.31, p<0.001)$, and (3) depressive symptoms $(\beta=0.16, p=0.01)$. When lying to parents was controlled for in hierarchical linear regressions, parenting by lying was directly associated with (1) stress $(\beta=0.25, p<0.001)$ and (2) anxiety $(\beta=0.27, p<0.001)$, but not depressive symptoms $(\beta=0.11, p=0.054)$.

Parent Relationships. The results show that parenting by lying in childhood was significantly associated with lower levels of (1) parent trust $(\beta=-0.20, p=0.002),(2)$ parent communication $(\beta=-0.12, p=0.048),(3)$ and parent overall relationship quality $(\beta=-0.19, p=$ 0.003), and higher levels of parent alienation $(\beta=0.21, p=0.001)$.

Mediating Relationships. To address hypothesis three, four double mediations were run for each of the three outcomes (depressive symptoms, anxiety, and stress) because of the four parent-child relationship quality variables. Although Figure 1 is the final model, it depicts the overall hypothesized model where parenting by lying was associated with lying to parents, which in turn was related to parent-child variables and finally the three outcomes. With a double mediation, three mediation pathways are possible. In Model one, parenting by lying is the only mediator to be estimated while in model two, the parent-child quality variable is the only mediator. In model three however, both mediators are estimated (Figure 1). Thus, twelve double mediation analyses were run with lying to parents (mediator 1) being constant but mediator 2 
varied by the four parent-child quality variables. All 12 analyses were run using SPSS Process Macros v3.1 and bootstrapping procedures [24], with summary results outlined on Table 2.

Given that all 12 meditations (i.e. with four different parent-child relationship quality variables $\mathrm{x}$ three different outcomes) were significant for the same model (model 3), we report only the three mediation results involving the fourth parent-child relationship quality (i.e. overall parent-child relationship quality variable), which was also the composite score. (Table 2).

Model three was significant for all estimated models, indicating that the double mediation model best fitted the data in explaining the mechanism through which parenting by lying was associated with stress, anxiety and depressive symptoms independently (see Figure 1). In summary, there were significant indirect effects of parenting by lying on stress, anxiety, and depressive symptoms, through lying to parents and each of the parent-child relationship quality variables contiguously (Table 2).

\section{Discussion}

We investigated the phenomenon called parenting by lying, as established by past research $[1-2,6]$, by examining its linkages with internalizing behaviors (stress responses, anxiety and depressive symptoms), as well as parent relationships (trust, communication, alienation, and overall relationship quality). First and similar to previous authors [6], parenting by lying in childhood was significantly and positively associated with lying to parents as adults. This finding builds on previous literature with a larger and more diverse sample. These results also support the social cognitive learning theory that proposes that children model behaviors of role models including parents [7].

Considering the first hypothesis, we found that parenting by lying and lying to parents were significantly and negatively associated with anxiety, stress and depressive symptoms, with depressive symptoms having the weakest linkage. We also found that participants who reported 
higher levels of parenting by lying in childhood were significantly more likely to report more maladaptive relationship patterns with their parents. In addition, those that experienced higher levels of parenting by lying were also less likely to trust and communicate with their parents and were more likely to experience alienation as young adults. These findings are in tandem with the two pioneering studies and support the linkages found as related to parent-child relationships [2, $6]$.

Finally, the indirect effects of parenting by lying on stress, anxiety, and depressive symptoms as separate models were significant only in the presence of the two mediators. As such the indirect relationships between parenting by lying and internalizing behaviors were impacted not only through lying to parents [6] but also through parent-child relationship quality variables (alienation, trust, communication). Interestingly, for all models, lying to parents as an independent variable was not significantly associated with any internalizing behavior outcomes, as seen in study by Santos et al. [6]. However, an examination of the correlations between lying to parents and the parent-child relationship quality variables showed moderate correlations between .37 and .42 suggesting that for this sample, the two groups of variables were relatively independent of each other. Thus, the indirect effect of lying to parents is more clearly seen in the presence of parent-child relationship quality. This finding supports numerous studies and parenting frameworks that show that parent-child relationships have a fundamental role in understanding the associations between parental behaviors and behavior of their offspring across the lifespan $[3,12,14,25-26]$. Longitudinal mediation analyses should be considered to further examine the relationships between parenting by lying, lying to parents, and adult functioning, and in what circumstances these pattern holds.

\section{Implications}


From this study, we learn that parenting by lying as a parenting strategy does have significant associations with later internalizing behaviors among young adults, and the mechanism of association may be through the quality of parent relationships and through lying to parents. Parents, counselors, and therapists should thus consider exploring the frequency of parenting by lying among children (especially children with problem behaviors), lying to parents, and the parent-child relationship quality during encounters including assessments, family or child counselling/therapy, and social skills training. Similarly, clinicians should consider exploring the relationship between childhood parenting strategies and quality of parent - adult child relations, and its potential influences on internalizing behaviors among young adults.

Secondly, based on previous studies and our findings, the positive relationship between parenting by lying and young adult behaviors (including maladaptive ones) is gaining ground. This is true also for the relationship between parenting by lying and lying to parents in young adulthood (as studied so far). Further research on these associations over time is needed, because although there may be positive benefits to current parenting by lying strategies in childhood (e.g., to shape behavior [2]), there could also be long-term negative associations we have been unaware of. The objective of this study, in this new area of research, is not to malign parents or their parenting strategies, but rather to shed light on potential unforeseen negative effects over time. This awareness could lead to exploration of alternative childhood parenting strategies that gain the same positive outcomes in childhood and in adulthood, while reducing potential contributions to negative long-term maladaptive behaviors.

We are in the beginning stages of understanding the mechanisms through which this seemingly benign parenting strategy (parenting by lying) can have long term impacts. Early studies such as this led to broader understanding of other parenting behaviors. For example, the 
groundbreaking work in understanding the specific types and effects of parenting styles [8], and the numerous studies thereafter, have helped researchers and practitioners identify maladaptive and protective parenting styles within different groups. The numerous studies have in turn led to the development of effective prevention/intervention programs that promote healthy parenting and healthier functioning of children, adolescents and young adults [11, 15, 26-29]. Similarly, we hope that in studying the mechanisms through which parenting by lying practices of childhood are related to behavioral and psychosocial adjustments of children in later years, more optimal parenting strategies that serve the same goals as current parenting by lying practices can be identified and promoted.

\section{Limitations and Future Research}

The present study has some limitations that serve as opportunities for further research. The cross-sectional and retrospective nature of this study and other published studies in this area limits the kinds of conclusions that can be drawn because of the temporal nature of the data. For example, primacy of ordering of constructs are not yet clear with cross-sectional mediations; as such only exploratory interpretations can be offered. Still, given the paucity of research in this area, such cross-sectional studies are important as they provide impetus for longitudinal studies to be designed and executed. Secondly, participants were asked questions about events in their childhood, which occurred approximately over a decade prior. Given what is known about the plasticity and unreliability of memories over time, participants could have forgotten that an occasion of parenting by lying occurred or the suggestion of lying in the study could have created a false memory. Yet, for now retrospective cross-sectional examinations are the main designs used in published works in this field. Future studies should consider longitudinal designs that include these measures. Besides a longitudinal design, future studies should also consider 
increasing the sample size and collecting data from adolescents and adults across different age groups.

Parenting by lying is a burgeoning area of study that has multiple potentials for future research since there are very limited studies so far. For example, culture influences parenting broadly and therefore, parenting by lying strategies. With limited studies that have examined the effects of parenting by lying among cultural groups [e.g. 2], future studies should examine these and other areas including individual variables such as the role of children's temperament, psychopathology and cultural dimensions. Also, age and other factors (such as trust, personality) could influence whether the child believes the lie, which in turn could affect the child's reaction to the lie and their psychosocial development. For example, if a child knows that a statement is a lie, they may instead find the statement amusing. Additionally, although previous studies have examined the frequency of various types of lies [1,2], this study examined parenting by lying in general. It is possible that different types of lies, such as lies designed to control emotions and behavior, could impact development differently. Future studies should also address these research questions in the quest to further and accurately understand the impact of parenting by lying in childhood on adult functioning. Lastly, since parents lie to their children for various reasons, further studies could examine how the goal/purpose for lying influences the types of lying used and their effects on child responses (short term) and adult functioning (long term).

\section{Summary}

In conclusion, this study is one of very few studies that have examined the associations between parenting by lying in childhood and adult functioning. While parenting is often rewarding and is an important endeavor, we sought to shed more light on possible pathways through which parenting by lying in childhood was associated with lying to parents, parent-child 
relationship quality, and internalizing behaviors in younghood (depressive, anxiety and stress symptoms). Results indicated that the relationship between parenting by lying in childhood and internalizing behaviors in adulthood was best explained when lying to parents and parent-child relationship quality were simultaneously examined as a double mediation. Although parenting by lying may not be intentionally used in childhood to harm; an awareness, however, of its potential and varied outcomes over time in the context of parent-child relationship quality is important to know. Such knowledge will empower parents, professionals and the community at large to refine and develop better/healthier parenting strategies.

\section{Data Availability Statement}

The data collection procedure and plans, in addition to other materials (e.g., output, IRB paperwork) can be found on https://osf.io/kp6ye/files/. Any other information may be requested from the corresponding author. 


\section{References}

1. Heyman GD, Luu DH, Lee K (2009) Parenting by lying. J Moral Educ 38(3): 353-369

2. Heyman GD, Hsu AS, Fu G, Lee K (2013) Instrumental lying by parents in the US and China. Int J Psychol, 48(6): 1176-1184

3. Murphey DA (1992) Constructing the child: Relations between parents' beliefs and child outcomes. Dev Rev, 12(2): 199-232

4. Roberts L, Marx JM, Musher-Eizenman DR (2018) Using food as a reward: An examination of parental reward practices. Appetite, 120: 318-326

5. Scaramella LV, Leve LD (2004) Clarifying parent-child reciprocities during early childhood: The early childhood coercion model. Clin Child Fam Psychol Rev, 7(2): 89107

6. Santos RM, Zanette S, Kwok SM, Heyman GD, Lee K (2017) Exposure to parenting by lying in childhood: Associations with negative outcomes in adulthood. Front Psychol, 8: 1-9

7. Bandura A (2001) Social cognitive theory: An agentic perspective. Annu Rev Psychol, 52(1): $1-26$

8. Baumrind D (1971) Current patterns of parental authority. Dev Psychol, 4(1, Pt.2): 1-103

9. Davis-Kean PE (2005) The influence of parent education and family income on child achievement: The indirect role of parental expectations and the home environment. J Fam Psychol, 19(2): 294-304

10. Elgar FJ, Mills RS, McGrath, PJ, Waschbusch DA, Brownridge DA (2007) Maternal and paternal depressive symptoms and child maladjustment: The mediating role of parental behavior. J Abnorm Child Psychol, 35(6): 943-955 
11. Lippold MA, Davis KD, Lawson KM, McHale SM (2016) Day-to-day consistency in positive parent-child interactions and youth well-being. J Child Fam Stud, 25(12): 35843592

12. Maccoby EE (1992) Trends in the study of socialization: Is there a Lewinian heritage? J Soc Issues, 48(2): 171-185

13. Lerner RM, Johnson SK, Buckingham MH (2015) Relational developmental systemsbased theories and the study of children and families: Lerner and Spanier (1978) revisited. J Fam Theory Rev, 7(2): 83-104

14. Malm EK, Henrich CC (2019) Longitudinal relationships between parent factors, children's bullying, and victimization behaviors. Child Psychiatry Hum Dev, 50: 789802

15. Bureau JS, Mageau GA (2014) Parental autonomy support and honesty: The mediating role of identification with the honesty value and perceived costs and benefits of honesty. J Adolesc, 37(3): 225-236

16. Cumsille P, Darling N, Martínez ML (2010) Shading the truth: The patterning of adolescents' decisions to avoid issues, disclose, or lie to parents. J Adolesc, 33(2): 285296

17. Engels RCME, Finkenauer C, van Kooten DC (2006) Lying behavior, family functioning and adjustment in early adolescence. J Youth Adolesc, 35: 949-958

18. Feiss, R., Dolinger, S. B., Merritt, M., Reiche, E., Martin, K., Yanes, J. A., Thomas, C. M., \& Pangelinan, M. (2019). A systematic review and meta-analysis of school-based stress, anxiety, and depression prevention programs for adolescents. J Youth Adolesc , 48(9): 1668-1685 
19. Gullone E, Robinson K (2005) The Inventory of Parent and Peer Attachment Revised (IPPA-R) for children: A psychometric investigation. Clin Psychol Psychother, 12(1): 6779

20. US Census (2012). 2010 Census of Population and Housing. https://www.census.gov/prod/cen2010/cph-2-19.pdf

21. Faul F, Erdfelder E, Buchner A, Lang AG (2009) Statistical power analyses using G*Power 3.1: Tests for correlation and regression analyses. Behav Res Methods, 41: $1149-1160$

22. Lovibond SH, Lovibond PF (1995) Manual for the Depression Anxiety \& Stress Scales. (2nd Ed.) Sydney: Psychology Foundation.

23. Armsden GC, Greenberg MT (1987) The inventory of parent and peer attachment: Individual differences and their relationship to psychological well-being in adolescence. $\mathrm{J}$ Youth Adolesc, 16: 427-454

24. Hayes AF (2018) Introduction to mediation, moderation, and conditional process analysis: a regression-based approach (2nd ed.). New York, NY: The Guilford Press.

25. Murray KW, Dwyer KM, Rubin KH, Knighton-Wisor S, Booth-LaForce C (2014). Parent-child relationships, parental psychological control, and aggression: Maternal and paternal relationships. J Youth Adolesc, 43(8): 1361-1373

26. Raudino A, Fergusson DM, Horwood LJ (2013) The quality of parent/child relationships in adolescence is associated with poor adult psychosocial adjustment. J Adolesc, 36(2): $331-340$ 
27. Pinquart M (2017a) Associations of parenting dimensions and styles with externalizing problems of children and adolescents: An updated meta-analysis. Dev Psychol, 53(5): 873

28. Pinquart M (2017b) Associations of parenting dimensions and styles with internalizing symptoms in children and adolescents: A meta-analysis. Marriage Fam Rev, 53(7): 613640

29. Gershoff ET, Grogan-Kaylor A (2016) Spanking and child outcomes: Old controversies and new meta-analyses. J Fam Psychol, 30(4): 453-469. 